\title{
Classificação Internacional de Práticas de Enfermagem em Saúde Coletiva - CIPESC ${ }^{\circledR}$
}

\author{
INTERNATIONAL CLASSIFICATION OF NURSING PRACTICES \\ IN COLLECTIVE HEALTH - CIPESC ${ }^{\circledR}$
}

\section{CLASIFICACIÓN DE LAS PRÁCTICAS DE ENFERMERÍA EN SALUD COLECTIVA - CIPESC ${ }^{\circledR}$}

\author{
Marcia Regina Cubas', Emiko Yoshikawa Egry²
}

\begin{abstract}
RESUMO
O projeto da Classificação Internacional da Prática de Enfermagem em Saúde Coletiva - CIPESC ${ }^{\circledR}$ foi a contribuição brasileira para incorporar a noção do coletivo na Classificação Internacional da Prática de Enfermagem $\mathrm{CIPE}^{\circledR}$. $\mathrm{O}$ artigo tem como objetivo analisar a produção bibliográfica acerca das classificações de enfermagem, disponíveis na base de dados da biblioteca virtual em saúde, a partir de 1990. Trata-se de uma pesquisa bibliográfica sobre os sistemas classificatórios das práticas de enfermagem, e que faz parte de uma pesquisa que pretende identificar a face coletiva do instrumento CIPESC $^{\circledR}$ na rede municipal de saúde de Curitiba-PR. Constatou-se que, referente aos sistemas classificatórios em geral, a maioria dos textos acessados baseia-se no modelo clínico-individual e os que enfatizam o modelo epidemiológico e social ainda é minoria. Além disso, a maioria dos estudos, com enfoque no coletivo, é divulgada pelas publicações da Associação Brasileira de Enfermagem, tornando-a hegemônica na disseminação dos conhecimentos acerca do tema, em sua face coletiva.
\end{abstract}

\section{DESCRITORES}

Classificação.

Enfermagem em saúde pública.

Enfermagem em saúde comunitária.

Diagnóstico de enfermagem.

Pesquisa em enfermagem.

\begin{abstract}
The project International Classification of the Nursing Practices in Collective Health - CIPESC ${ }^{\circledR}$ was the Brazilian contribution for the incorporation of the notion of collectiveness in the International Classification of Nursing Practice - ICNP ${ }^{\circledR}$. This article aims at analyzing the bibliographical production concerning nursing classifications available in the virtual library in health database since 1990. This is a bibliographical survey of nursing practice classification systems and is part of a larger research that intends to identify the collective face of the CIPESC ${ }^{\circ}$ instrument in the municipal health network of the city of Curitiba, in the State of Paraná. The result shows that the majority of the texts are based on the clinical-individual model, and those that emphasize the social-epidemiologic model are still a minority. In addition, most studies focused on the collective were disseminated through the publications of the Brazilian Nursing Association, which has the hegemony in the diffusion of the knowledge about the collective face of classification systems.
\end{abstract}

\section{RESUMEN}

El proyecto de Clasificación Internacional de la Práctica de Enfermería en Salud Colectiva - CIPESC ${ }^{\circledR}$ fue la contribución brasileña para incorporar la noción del colectivo en la Clasificación Internacional de la Práctica de Enfermería CIPE ${ }^{\circledR}$. EI artículo tiene como objetivo analizar la producción bibliográfica acerca de las clasificaciones de enfermería en bases de informaciones disponibles en la biblioteca virtual en salud desde 1990 . Se trata de una investigación bibliográfica acerca de los sistemas clasificatorios y es parte de un proyecto que pretende identificar la faz colectiva del instrumento CIPESC $^{\circledR}$ utilizado en la red municipal de salud de Curitiba, capital del estado de Paraná. La mayoría de los artículos referentes a los sistemas clasificatorios en general, son los que se asientan en el modelo clínico-individual, sendo una minoría los que se asientan en el modelo epidemiológico social. Aún, la mayoría de los estudios, con enfoque en el colectivo están siendo divulgados por las publicaciones de la Asociación Brasileña de Enfermería, volviéndola hegemónica en la diseminación de los conocimientos a cerca del tema, en su faz colectiva.

\author{
KEY WORDS \\ Classification. \\ Public health nursing. \\ Community health nursing. \\ Nursing diagnosis. \\ Nursing research.
}

\author{
DESCRIPTORES \\ Clasificación. \\ Enfermería en salud pública. \\ Enfermería en salud comunitaria. \\ Diagnóstico de enfermería. \\ Investigación en enfermería.
} 1 Enfermeira. Mestre em Saúde Pública. Doutoranda em Enfermagem da Escola de Enfermagem, Universidade de São Paulo (EEUSP). Professora
Assistente da Pontifícia Universidade Católica do Paraná. Curitiba, PR, Brasil. m.cubas@pucpr.br ${ }^{2}$ Enfermeira. Professora Titular do Departamento de Enfermagem em Saúde Coletiva, Escola de Enfermagem, Universidade de São Paulo (EEUSP). São Paulo, SP, Brasil. emiyegry@usp.br 


\section{INTRODUÇ̃̃O}

Diante da constatação de que os sistemas de classificação de enfermagem utilizados mundialmente evidenciavam um direcionamento à área hospitalar, o Conselho Internacional de Enfermeiros - CIE, decidiu orientar um projeto internacional voltado para a extra-internação. A Associação Brasileira de Enfermagem - ABEn assumiu o compromisso de desenvolver o projeto no País e, em 1996, promoveu a primeira oficina de trabalho que deu origem ao projeto Classificação Internacional de Práticas de Enfermagem em Saúde Coletiva - CIPESC, contribuição brasileira à Classificação Internacional de Práticas de Enfermagem -CIPE ${ }^{\circledR(1)}$.

O referido projeto ancorava-se nos princípios do SUS - Sistema Único de Saúde e seus propósitos centrais eram:

\begin{abstract}
Estabelecer mecanismos de cooperação para a classificação da prática de enfermagem em saúde coletiva no País; Revistar as práticas de enfermagem em saúde coletiva no País, contextualizadas no processo de produção em saúde, diante da implantação do Sistema Único de Saúde; Construir um sistema de informação das práticas de enfermagem em saúde coletiva que permitam a sua classificação, troca de experiências e interlocução nos níveis nacional e internacional(1).
\end{abstract}

O referencial teórico-metodológico do projeto explicitava a necessidade do repensar crítico acerca do processo de trabalho da enfermagem brasileira em seus elementos constitutivos: objeto, finalidade e instrumentos/ meios, articulados dialeticamente. Articulação esta que se realiza

mergulhada no saber-ideológico que rege o processo de trabalho, ou seja, teleologicamente posto pelas políticas que regulam a produção em saúde de dada época e de dada sociedade ${ }^{(2)}$.

Sendo assim, a Classificação das Práticas de Enfermagem é entendida como uma totalidade-parte do trabaIho da enfermagem e deve também ser aproximada, em sua concepção teórico-conceitual, pela totalidade-parte do processo de produção em saúde em suas diferentes dimensões ${ }^{(2)}$

Em 1998, já havia um resultado do projeto CIPESC $^{\circledR}$ a ser compartilhado e que permitiu novas leituras e análises, propiciando então, a geração do $\operatorname{CIPESC}^{\circledR}$ - ABEn fase $2^{(3)}$ com o intuito de dar continuidade e contribuir para a efetiva aplicação dos resultados da primeira fase.

Em face desta possibilidade, em 2001, a ABEn Seção Paraná apresentou uma tese na VI Conferência Municipal de Saúde (2001) de Curitiba-PR, aprovada na sua integralidade, propondo a construção da Rede de Atenção Contínua de Enfermagem no Sistema Integrado de Serviços de Saúde com estratégias voltadas para: a) inclusão de todas as atividades desempenhadas pelo profissional Enfermeiro no relatório de produtividade; b) criação de um campo específico para o registro da Enfermagem no prontuário eletrônico único do paciente; c) estabelecimento de parcerias com instituições de ensino para capacitação da força de trabalho; e d) consulta de Enfermagem integrada às programações locais ${ }^{(4)}$. Neste mesmo ano, impulsionado pela necessidade de adensar os conhecimentos acerca dos marcos teóricos das práticas de enfermagem, ocorreu um movimento de revisão das práticas instituídas, antecedendo a sua inclusão no prontuário eletrônico. Esta revisão realizada à luz de novos paradigmas, possibilitou a implantação da CIPE $^{\circledR} /$ CIPESC $^{\circledR}$ no sistema informatizado da Secretaria Municipal da Saúde de Curitiba - SMS ${ }^{(5)}$.

Nos últimos três anos, uma equipe de trabalho constituída de um grupo condutor com seis enfermeiros, um grande grupo de trabalho com cerca de cinqüenta enfermeiros e a assessoria da ABEn - nacional, dedicou-se ao estudo e à implantação dos elementos da prática de enfermagem no prontuário eletrônico da SMS de Curitiba, Paraná. Em junho de 2004 foi oficializada a implantação municipal da CIPESC $^{\circledR}$ iniciando-se na área da Saúde da Mulher, em 2005 incorporou a de Saúde da Criança e, em 2006, após um movimento contínuo de avaliação, implementará a nomenclatura com entrada pelas Necessidades Humanas Básicas ${ }^{(6)}$.

É neste cenário de implementação que surgem as questões desta revisão: como está o estado da arte do conhecimento acerca da classificação das práticas de enfermagem em saúde coletiva? Há experiências no Brasil que tenham desdobrado o projeto CIPESC?

Do ponto de vista do materialismo histórico e dialético, os sistemas de classificação podem ser vistos como meios ou instrumentos do processo de trabalho de enfermeiro. Nesta perspectiva, os meios são distinguidos em: a) meios de sentido estrito que são os que servem de intermediário entre o trabalhador e o objeto e b) meios de sentido amplo que agregam todas as condições que são indispensáveis à realização do processo de transformação. Sendo o primeiro o mais significativo, pois revela o tipo de trabalho a ser realizado, conseqüentemente o tipo de relação entre o trabalhador e os meios de produção(7).

CIPESC pode ser pensada enquanto instrumento do processo de trabalho assistencial do enfermeiro, mas não só. Ela possibilita visibilizar as estruturas maiores que organizam o trabalho da enfermagem, ao mesmo tempo, podem se tornar um poderoso instrumento potencializador da avaliação processual dos resultados, benefícios e impactos da ação da enfermagem. Não sendo um mero sistema inflexível no qual a realidade precisa se amoldar à teoria classificatória, ela permite a captura dos padrões diferenciados de práticas de enfermagem, que no nosso País se encontram multifacetadas e realizados por multiagentes. Assim pensado, a CIPESC ${ }^{\circledR}$ é um potente instrumento de trabalho do enfermeiro em saúde coletiva, tanto do ponto de vista assistencial, quanto de gerência e de investigação. 
Trata-se neste artigo de conhecer e analisar a produção científica acerca das classificações de enfermagem, em especial a produção referenciada a CIPESC ${ }^{\circledR}$ em bases de dados disponíveis na Biblioteca Virtual em Saúde.

\section{MÉTODO}

Utilizou-se pesquisa bibliográfica nas bases de dados disponíveis na Biblioteca Virtual em Saúde, realizada em 2005 e estabeleceu-se o recorte de tempo das publicações de 1990 a 2005, por ser o período em que surgiram as publicações acerca dos sistemas classificatórios das práticas de enfermagem em saúde coletiva. O método foi levantamento e revisão das literaturas especializadas mais importantes publicadas a respeito do tema ${ }^{(8)}$, que foram organizadas de acordo com o tipo de sistema classificatório estudado, a face do objeto da assistência (individual ou coletivo) e o cenário de aplicação (intra ou extrainternação). Foram utilizados os seguintes descritores: Sistematização and saúde pública; Sistematização and classificação and enfermagem; Sistematização or classificação and enfermagem; CIPESC; Diagnóstico and enfermagem. Após busca inicial, o material foi organizado de forma a excluir textos que não se referiam à enfermagem.

Ao acessar os textos nas bases de dados, também foram excluídos os que se repetiam em diferentes descritores e nos distintos bancos. Em relação aos descritores: sistematização or classificação and enfermagem e diagnóstico and enfermagem verificou-se que as Bases de Dados da Enfermagem - BDENF continham todas as referências encontradas nas bases Lilacs, Adolec e Medline e, para não haver repetições serão citadas apenas na coluna do BDENF.

\section{RESULTADOS E DISCUSSÕES}

A busca dos artigos nas bases de dados através dos descritores selecionados resultou em 179 textos divulgados, sendo que a maioria encontrava-se alojado no BDENF e dentro do descritor sistematização or classificação and enfermagem, conforme pode-se verificar na Tabela 1.

Tabela 1 - Distribuição dos artigos referentes à classificação em enfermagem constantes nas bases de dados, de acordo com os descritores, Biblioteca Regional de Medicina (BIREME) -São Paulo - 2005

\begin{tabular}{|c|c|c|c|c|c|c|}
\hline Base de dados & Lilacs & Medline & Adolec & BDENF & OPAS & Total \\
\hline Sistematização and saúde pública & -- & -- & -- & -- & -- & -- \\
\hline $\begin{array}{l}\text { Sistematização and classificação } \\
\text { and enfermagem }\end{array}$ & 27 & 22 & 2 & 7 & 1 & 59 \\
\hline Sistematização or classificação and enfermagem & -- & -- & -- & 73 & -- & 73 \\
\hline Cipesc & 5 & 5 & -- & -- & 1 & 11 \\
\hline Total geral & 32 & 27 & 2 & 116 & 2 & 179 \\
\hline
\end{tabular}

A combinação de descritores sistematização e saúde pública não foi encontrada nas bases consultadas, todas as demais combinações de descritores apareceram em pelo menos uma das bases.

A Figura 1 apresenta a distribuição percentual das publicações conforme o tipo de pesquisa, que foi feita segundo a classificação indicada no periódico em que foi publicado. Além disso, os artigos resultantes de dissertações ou teses foram classificados à parte das outras pesquisas que não eram oriundas de trabalhos de pós-graduação. Ressalta-se que as produções científicas tipificadas como pesquisa, quer seja derivada ou não de teses ou dissertações, representaram a maioria $(63,15 \%)$ dos estudos encontrados nas bases de dados.

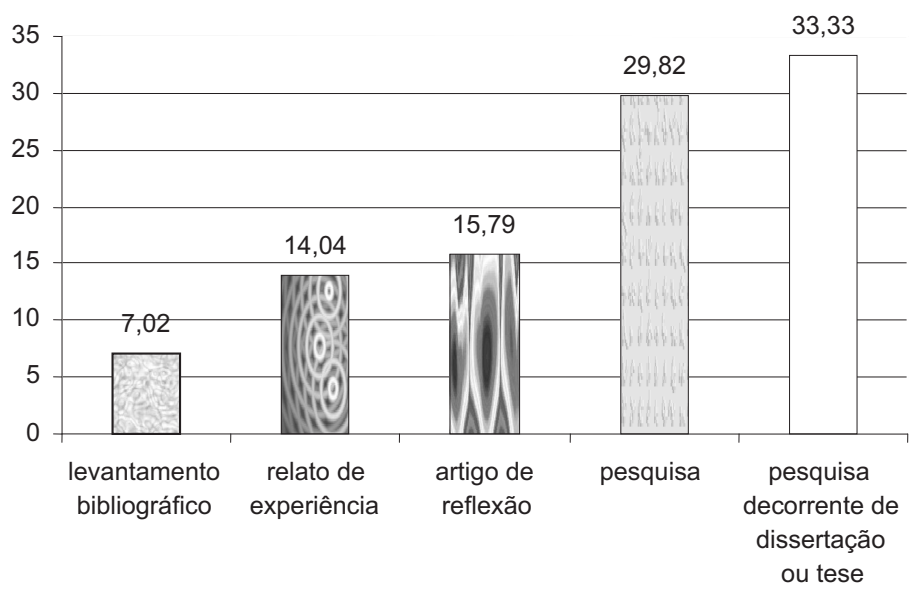

Figura 1 - Distribuição das publicações referentes à classificação em enfermagem encontradas na Bireme, segundo o tipo de pesquisa, Biblioteca Regional de Medicina (BIREME) - São Paulo -2005 
Estudos sobre a sistematização dos cuidados de enfermagem nas diferentes fases do ciclo de vida do indivíduo somados aos relativos à sistematização dos cuidados de enfermagem a grupo de indivíduos portadores de doenças crônico-degenerativas representaram $25 \%$ dos objetos de estudo. A CIPESC ${ }^{\circledR}$ foi objeto de estudo em menos de $10 \%$ das publicações encontradas. Os estudos que realizam validações de diagnósticos e de termos representaram $13,84 \%$ das pesquisas que se relacionavam diretamente a sistemas classificatórios - NANDA ${ }^{\circledR}$ e CIPE $^{\circledR} /$ CIPESC $^{\circledR}$.

A análise das fontes referenciadas na busca sobre sistematização e classificação e enfermagem, permitiu verificar o reflexo do modelo hegemônico assistencial: a maioria estava relacionada à área hospitalar e com a inserção da tecnologia, ou seja, estudos que se dizem respeito ao uso de ferramentas computacionais. A classificação NANDA ${ }^{\circledR}$ - North American Nursing Diagnosis Association, apareceu em grande parte dos trabalhos, inclusive nas teses, bem como sua relação com CID- $10^{\circledR}$ - Classificação Internacional das Doenças. A CIPESC ${ }^{\circledR}$ aparece apenas em três: uma tese usando o material produzido pelo primeiro projeto da ABEn recortada no tema processo de trabalho gerencial e dois artigos que discutem a adaptação transcultural da $\mathrm{CIPE}^{\circledR}$ e CIPESC ${ }^{\circledR}$, estas mesmas publicações estão também disponibilizadas na busca do descritor cipesc.

Uma das publicações que pesquisou a percepção dos enfermeiros hospitalares quanto à necessidade de registro da enfermagem, revelou um conhecimento incipiente sobre os sistemas de informação, bem como o pouco desejo de utilizar um sistema de classificação em todas as fases do processo de enfermagem ${ }^{(9)}$. Essa pequena importância dada ao assunto, poderá perpetuar as dificuldades na construção do saber da enfermagem, devido às inconsistências conceituais, termos vagos e ambíguos na denominação das ações e dos fenômenos que envolvem a profissão(10).

Um resumo publicado no Reino Unido chamou a atenção pelo fato do autor apontar para a importância da inclusão, nos sistemas classificatórios, de termos que contenham a cultura própria das enfermeiras britâni$\operatorname{cas}^{(11)}$, concluindo que uma boa classificação internacional deverá prever as diferentes inserções do cuidado nas distintas sociedades.

Nos setenta e três resultados encontrados sob os descritores sistematização ou classificação e enfermagem foram realçados quarenta e dois textos que versavam sobre $o$ atendimento do enfermeiro na saúde coletiva ou classificações de diagnóstico e dentre eles não houve nenhum referente à CIPESC ${ }^{\circledR}$ e três estudos utilizaram a taxonomia NANDA ${ }^{\circledR}$. O que se observou é que a assistência de enfermagem está direcionada, mesmo na saúde coletiva, à sistematização do atendimento individual, voltada à atenção a grupos prioritários com o enfoque de risco biológico: hipertensos, diabéticos, crianças em cre- ches, entre outros. Confirma isto o estudo sobre a pesquisa nacional da CIPESC ${ }^{\circledR}$, quando mesmo em se tratando de objetos grupais ou coletivos, estes se encontram submetidos a recortes cronológicos, por patologias ou lugares nos quais se dão a assistência, a exemplo de creches, escolas, entre outros ${ }^{(4)}$.

Nas referências sobre a cipesc observou-se que o produto do trabalho da $A B E n$ ainda não alcançou a plenitude de suas possibilidades. As referências têm como base o material produzido pelo grupo de pesquisadores do projeto CIPESC de 1996 a 2000, disponíveis na Série Didática: Enfermagem no SUS (www.abennacional.org.br) e em artigos que se referem a estes trabalhos, ou seja, há muito a ser pesquisado e escrito sobre a experiência.

As publicações da Série Didática não constam na sua totalidade neste descritor, sendo parte delas acessadas nos outros descritores. Ressalte-se que, apenas um dentre os dezesseis cenários estudados pelo primeiro projeto CIPESC - o de Porto Alegre ${ }^{(12)}$ - encontra-se publicado em periódico indexado, tornando os demais invisíveis às buscas através do descritor cipesc. Se os pesquisadores não buscarem diretamente na base de publicações da Associação Brasileira de Enfermagem, a imensa produção da pesquisa CIPESC realizada pela ABEn permanecerá escondida, pois elas não se encontram nas bases correntes de dados. Torna-se importante, pois, repensar nestas formas de disseminação das produções científicas.

Por fim a busca de informações em bases dos dados através dos descritores diagnóstico e enfermagem resultou na seleção de 36 referências. A autora de um dos textos escolhidos compreendia a taxonomia de diagnóstico e prescrição como instrumentos de trabalho do enfermeiro e conseqüentemente com possibilidade de maior definição do objeto de trabalho da enfermagem ${ }^{(13)}$, um outro texto estudou a relação entre o diagnóstico e os elementos do processo de trabalho do enfermeiro ${ }^{(14)}$, porém ambos direcionados a área hospitalar e ao atendimento clínico e individualizado. Novamente não houve citação da CIPESC $^{\circledR}$, tendo sido a taxonomia NANDA ${ }^{\circledR}$ a preponderante em todas as publicações.

Nesta busca, dois estudos contribuíram para o resgate histórico dos sistemas de classificação: um deles, demonstrou a evolução do movimento das definições que pretendem explicar o diagnóstico de enfermagem ${ }^{(15)}$, e o segundo, que apresentou os diagnósticos como um desafio para os anos $1990^{(16)}$.

Os resultados apresentados nas das publicações, ao menos em parte delas, buscam orientar linhas de condutas sistematizadas para o cuidado de enfermagem, vinculadas ao uso de algum tipo de sistema de classificação. Observa-se que em cerca de $25 \%$ das divulgações, os autores apontam para a importância de sistemas de classificação como subsídio ao cuidado de enfermagem, assim como para a importância da sistematização da assistência ligada ao modelo assistencial. 


\section{CONSIDERAÇÕES FINAIS}

A busca de informações nas bases de dados pode parecer uma tarefa solitária. No entanto, as sucessivas aproximações ao objeto recortado e as leituras decorrentes desta busca ajudam a inserir o pesquisador num mundo de questionamentos acerca deste mesmo objeto. Neste ponto é tarefa coletiva, descobrem-se lacunas, consistências e inconsistências na literatura ${ }^{(7)}$ e desvela-se possibilidades de intervenções na prática.

Em relação ao objeto recortado, ou seja a $\mathrm{CIPESC}^{\circledR}$, como instrumento do processo de trabalho do enfermeiro em saúde coletiva - muito se tem a descobrir e pesquisar. Os artigos encontrados estão voltados mais ao modelo hegemônico clínico-individual, sendo que a face coletiva ainda é pouco estudada, principalmente no âmbito dos sistemas classificatórios. Para impacto no coletivo, entretanto, faz-se necessária a mudança de paradigmas, buscando a interpretação do processo saúde-doença articulado com os seus determinantes e com as necessidades sociais, indo além do modelo voltado para as necessidades humanas básicas e intervenções sobre os valores vitais, biológicos e psicológicos, resultando numa forma de assistir cada vez mais totalizadora ${ }^{(17)}$.

Os resultados apontam para a grande contribuição da ABEn na divulgação dos conhecimentos acerca da classi-

\section{REFERÊNCIAS}

1. Silva IA, Egry EY, Sena RR, Almeida MCP, Antunes MJM. A participação do Brasil no projeto de Classificação Internacional das Práticas de Enfermagem. In: Fonseca RMGS, Bertolozzi MR, Silva IA. O Uso da epidemiologia social na enfermagem de saúde coletiva. Brasília: ABEn; 1997. p. 65-81.

2. Egry EY. A classificação da prática de enfermagem como instrumento do cuidado de enfermagem na perspectiva individual e coletiva: limites e possibilidades. In: Anais do $50^{\circ}$ Congresso Brasileiro de Enfermagem; 1998; Salvador, BA, Brasil. Salvador: ABEn-Seção-BA; 1999. p. 80-7.

3. Antunes MJM. A prática de enfermagem e os sistemas de classificação: a ótica da ABEn. In: Garcia TR, Nóbrega, MML. Sistemas de Classificação das Práticas de Enfermagem: um trabalho coletivo. João Pessoa: ABEn/Idéia; 2000. p. 9-18.

4. Curitiba. Conselho Municipal de Saúde. Relatório da 6으 Conferência Municipal de Saúde: Plano Municipal de Saúde de Curitiba - 2002-2005. Curitiba; 2001.

5. Albuquerque LM, Cubas MR, Lopes MGD, Zagonel IPS. Diagnóstico de enfermagem na transição de paradigmas da prática profissional. In: Livro programa do 6ㅇ Simpósio Nacional de Diagnóstico de Enfermagem e 1은 Simpósio Internacional sobre Classificações de Enfermagem; 2002 ago. 7-9; São Paulo, SP, Brasil. São Paulo: ABEn-Seção-SP; 2002. p. 27. ficação das práticas de enfermagem no âmbito da saúde coletiva, tendo sido o mais importante veículo disseminador da produção acerca do tema. Entretanto, isto não se expressa nas bases de dados mais amplos, ou seja, os leitores e pesquisadores que não conhecem a contribuição específica da ABEn no Projeto CIPESC e não conhecem o site da Associação, dificilmente conseguirão ter idéia da totalidade desta importante produção.

No intuito de buscar não deixar hiato entre o conhecimento necessário para intervenção e o conhecimento que se possui... ${ }^{(18)}$, certamente há que mostrar a produção da ABEn no conjunto, composta por oito publicações disponibilizadas no site www.abennacional.org.br.

Além disso, há outros relatos importantes, tais como as teses e dissertações produzidas na Escola de Enfermagem da USP e na Universidade Federal do Paraná decorrentes dos estudos da CIPESC ${ }^{\circledR}$ que ainda não são encontrados em bases de dados. Mais recentemente, uma experiência nacional que visa sistematizar as práticas da enfermagem no âmbito da extra-internação foi retratada no artigo Avaliação da implantação do CIPESC $^{\circledR}$ em Curitiba ${ }^{(19)}$ em que autoras referem os sistemas de classificação das práticas como projetos emancipatórios da profissão, pois possibilitam visibilizar as práticas da enfermagem, refletir sobre o cotidiano do cuidado, quer na face individual, quer na do coletivo.

6. Albuquerque LM, Cubas MR, Martins SK. Nomenclatura de diagnósticos e intervenções de enfermagem da rede básica de saúde do município de Curitiba. In: Albuquerque LM, Cubas MR, organizadoras. Cipescando em Curitiba: construção e implementação de diagnósticos e intervenções de enfermagem na rede básica de saúde. Curitiba: ABEn-Seção-PR; 2005.

7. Castellanos BEP. O trabalho do enfermeiro a procura e encontro de um caminho para o seu estudo: da abordagem mecânico-funcionalista à pesquisa emancipatória [tese]. São Paulo: Escola de Enfermagem, Universidade de São Paulo; 1987.

8. LoBiondo-Wood G, Haber J. Pesquisa em enfermagem: métodos, avaliação crítica e utilização. 4a ed. Rio de Janeiro: Guanabara-Koogan; 2001.

9. Santos SR, Paula AFA, Lima JP. O enfermeiro e sua percepção sobre o sistema manual de registro no prontuário. Rev Lat Am Enferm. 2003;11(1):80-7.

10. Souza MF. As classificações e a construção de conhecimento na enfermagem. Rev Bras Enferm. 2002; 55(6): 691-6.

11. Hogston R. Nursing diagnosis and classification systems: a position paper. J Adv Nurs. 1997;26(3):496-500. 
12. Witt RR, Girardi MA, Malerba H, Fengler KP. Projeto CIPESCBrasil: caracterização da enfermagem no cenário Porto Alegre. Rev Gaúcha Enferm. 2002;23(1):103-13.

13. Cruz ICF. Diagnóstico prescrições de enfermagem recriando os instrumentos de trabalho. Texto Contexto Enferm. 1995;4(1):160-9.

14. Crossetti MGO. Algumas reflexões sobre o diagnóstico de enfermagem e sua relação com os elementos do processo de trabalho. Texto Contexto Enferm. 1995; $4(1): 150-9$.

15. Cruz DALM. Diagnóstico de enfermagem: aspectos históricos e definição. Rev Paul Enferm. 1994;13(1/3):3-7.
16. Perez VLAB, Nóbrega MML, Farias JN, Coler MS. Diagnóstico de enfermagem: um desafio para enfermagem para os anos 90. Rev Bras Enferm. 1990;43(1/4):14-8.

17. Oliveira MAC, Egry EY. A historicidade das teorias interpretativas do processo saúde-doença. Rev Esc Enferm USP. 2000;34(1):9-15.

18. Egry EY. Saúde coletiva construindo um novo método em enfermagem. São Paulo: Ícone; 1996.

19. Cubas MR, Albuquerque LM, Martins SK, Nóbrega MML. Avaliação da implantação do CIPESCÒ em Curitiba. Rev Esc Enferm USP. 2006;40(2):269-73. 